\title{
Fibers and Conductive Films Using Silver Nanoparticles and Nanowires by Near-Field Electrospinning Process
}

\author{
Cheng-Tang Pan, ${ }^{1}$ Tsung-Lin Yang, ${ }^{1}$ Yi-Chian Chen, ${ }^{1}$ Cherng-Yuh Su, ${ }^{2}$ \\ Shin-Pon Ju, ${ }^{1}$ Kun-Hao Hung, ${ }^{1}$ I-Chou Wu, ${ }^{1}$ Ci-Chang Hsieh, ${ }^{3}$ and Sheng-Chih Shen ${ }^{4}$ \\ ${ }^{1}$ Department of Mechanical and Electro-Mechanical Engineering, National Sun Yat-Sen University, Kaohsiung 80424, Taiwan \\ ${ }^{2}$ Institute of Manufacturing Technology, National Taipei University of Technology, Taipei 10608, Taiwan \\ ${ }^{3}$ Department of the Mechanical and Automation Engineering, National Kaohsiung First University of Science and Technology, \\ Kaohsiung 811, Taiwan \\ ${ }^{4}$ Department of Systems and Naval Mechatronic Engineering, National Cheng Kung University, Tainan 701, Taiwan
}

Correspondence should be addressed to Sheng-Chih Shen; scshen@mail.ncku.edu.tw

Received 4 September 2014; Accepted 17 March 2015

Academic Editor: Vladimir Sivakov

Copyright (C) 2015 Cheng-Tang Pan et al. This is an open access article distributed under the Creative Commons Attribution License, which permits unrestricted use, distribution, and reproduction in any medium, provided the original work is properly cited.

\begin{abstract}
The silver nanowires (AgNWs) and silver nanoparticles (AgNPs) were synthesized. With near-field electrospinning (NFES) process, fibers and thin films with AgNPs and AgNWs were fabricated. In the NFES process, $10 \mathrm{k}$ voltage was applied and the AgNPs and AgNWs fibers can be directly orderly collected without breaking and bending. Then, the characteristics of the fibers were analyzed by four-point probe and EDS. The conductive film was analyzed. When the thickness of films with AgNWs and AgNPs was $1.6 \mu \mathrm{m}$, the sheet resistance of films was $0.032 \Omega$ /sq which was superior to that of the commercial ITO. The transmissivity of films was analyzed. The transmissivity was inversely proportional to sheet resistance of the films. In the future, the fibers and films can be used as transparent conductive electrodes.
\end{abstract}

\section{Introduction}

Recently, as the smart phone technology developed rapidly, a lot of attention was focused on the application of transparent electrodes [1], which can be widely applied to the organic light-emitting diode (OLED) [2], touch screen [3], and thin film silicon solar cells. Currently, the prevailing material of the current transparent electrode was indium tin oxide (ITO) [4]. However, the disadvantages of ITO including expensive price, brittle material, and complicated process in the vacuum environment [5] resulted in extremely intensive energy and economically expensive during thin film fabrication process. Therefore, there were some emerging alternatives, such as graphene [6], silver nanoparticles (AgNPs) synthesized by chemical method [7], and silver nanowires (AgNWs) [8] with high conductivity and transmissivity used to replace ITO. The AgNWs were a promising alternative. Moreover, the farfield electrostatic spraying system was a technique which adopted high electric field to produce nonwoven materials that were excellent in the porosity, high surface area, fineness, and uniformity of the fibers [9]. Due to the necessity of a high applied voltage, the near-field electrospinning (NFES) process was developed. The fibers could be fabricated with low applied voltage. The direct-written polyethylene oxide (PEO) fibers fabricated by the NFES process were reported by Sun et al. [10]. In this study, the AgNWs and AgNPs were synthesized. With the NFES process, the suitable electric field was applied to electrospin the AgNPs fibers. Then, the AgNPs fibers were orderly collected. The characteristics of fibers were analyzed by Scanning Electron Microscope (SEM) and Energy Dispersive X-Ray (EDS). Next, the AgNWs were used to fabricate AgNWs film. The optical transmissivity and sheet resistance of AgNWs film were measured by $\mathrm{N} \& \mathrm{~K}$ analyzer and four-point probe.

\section{Experiment}

2.1. Electrospinning Process for AgNPs Fibers and Pure AgNWs. For the electrospinning process, the AgNPs sol gel was filled 


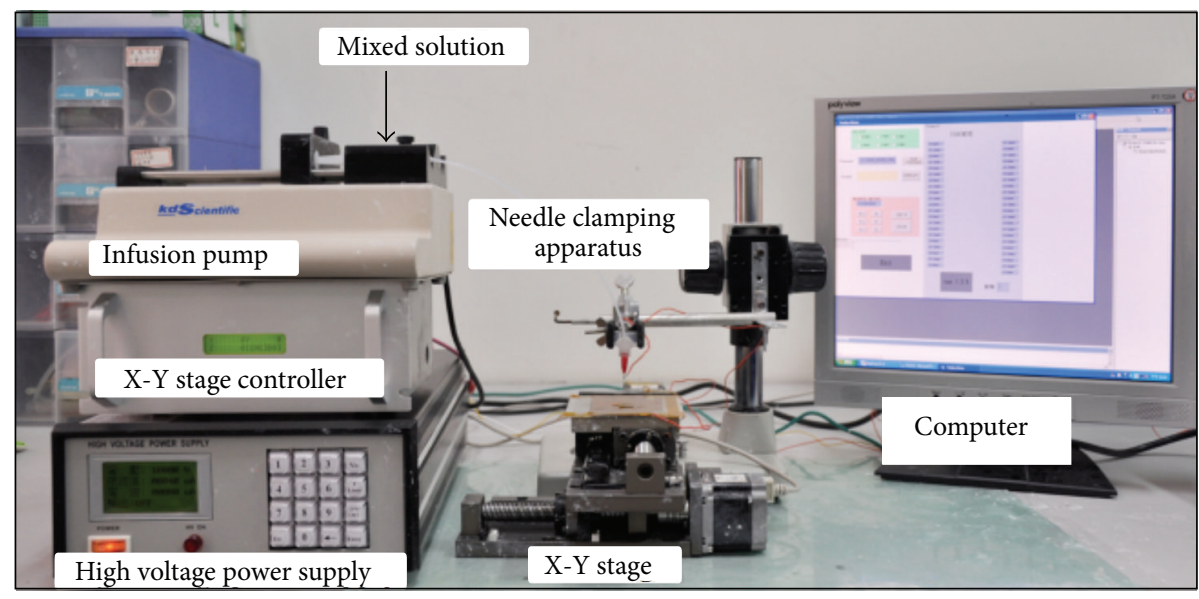

FIgURE 1: The setup of near-field electrospinning system.

in a syringe linked with a $0.5 \mathrm{~mm}$ diameter metallic needle to make the hemispherical solution appear at the tip of metallic needle. An electrode of a high voltage power supply was also attached to the tip of metallic needle. Thus, there was electric field created between hemispherical solution and collector. The applied electric field could make the positive ions be accumulated on the surface of hemispherical solution because of the electrostatic repulsion. When the repulsion was larger than the surface tension of hemispherical solution, the Taylor cone could be formed at the tip of hemispherical solution. Then, the repulsion could break through the solution and make the solution be ejected to form electrospun fibers. Therefore, the suitable applied voltage was necessary. In this study, it was set as $10 \mathrm{kV}$ for this experiment. Next, the syringe was horizontally installed on the syringe pump. The flow rate of solution was set as $10 \mu \mathrm{L} / \mathrm{h}$. The tip-to-collector distance was set as $3 \mathrm{~mm}$. The silicon or glass collector was put on the XY-table controlled by the programmable XY-table system. Figure 1 shows the NFES equipment. The droplet was dragged out by the electrical field to electrospin the AgNPs fibers.

Next, the polyol reduction process was adopted to synthesize AgNWs solution and then the solution was centrifuged. The acetone was added into AgNWs solution and the proportion of acetone to AgNWs solution was set as $2: 1$. The mixed solution was evenly stirred to make the AgNWs be precipitated at the bottom of the solution. Then, the acetone and ethylene glycol at the top of the solution were removed due to the fact that the ethylene glycol was just the solvent and reducing agent for the synthesis of AgNWs. The residual AgNWs were added in the centrifuge tubes and then deionized water was injected in the tubes to remove PVP. During the synthesis process, the PVP was the material in which oxygen bond could combine with $\mathrm{Ag}$ bond of silver and make silver grow anisotropically. However, the PVP could coat the surface of silver and make the silver nonconductive. In addition, the deionized water was a good solvent for dissolving PVP. The deionized water was adopted to remove PVP. The residual AgNWs with deionized water in the centrifuge tubes were centrifuged at $6000 \mathrm{rpm}$ for thirty minutes. The experimental process of injection of deionized water and centrifuging was repeated $3 \sim 5$ times until the above solution became transparent. The transparent solution in which the PVP dissolved was removed. The residual product in the centrifuge tube was the pure AgNWs. Finally, the pure AgNWs were added into deionized water and the solution was shaken to make pure AgNWs be dispersed evenly.

\section{Results and Discussion}

3.1. Results of Synthesis of PVP/AgNWs, AgNPs, Electrospun AgNPs Fiber, and Pure AgNWs. Figure 2(a) shows the formulated AgNWs solution. The AgNWs could not be observed clearly in the synthesized solution due to the fact that AgNWs were coated with PVP and the solution was full of thick ethylene glycol. Then, the AgNWs solution was centrifuged to obtain pure AgNWs. Figure 2(b) shows the clear pure AgNWs. The diameter and length of AgNWs were in the ranges $10 \sim 50 \mathrm{~nm}$ and $15 \sim 30 \mu \mathrm{m}$, respectively. Furthermore, Figure 2(c) shows the synthesized AgNPs which were accumulated together because of the cohesive force between AgNPs. During the NFES process, a suitable applied voltage was $10 \mathrm{kV}$. The moving path of collector on the XY-table was set as straight line. From the result of electrospinning process, Figure 3 shows that the electrospun AgNPs fiber was straight without breaking and bending. Therefore, the path of electrospun fiber could be controlled.

3.2. EDS Measurement. Figure 4 shows the result of EDS analysis. The Au element was detected due to the coating for SEM observation, and the Si and Ag elements were detected because of the silicon collector and AgNPs. Figure 4 shows the analysis of AgNPs solution. The carbon peak was high due to the fact that AgNPs were not centrifuged and the PVP which was a polymer was left behind on the surface of AgNPs. For the centrifuged AgNWs solution, the PVP was almost removed. There were just few residual PVP on the surface of AgNWs and the thickness of PVP was less than $2 \mathrm{~nm}$. Therefore, the carbon peak was very low in the EDS analysis of centrifuged AgNWs solution. 


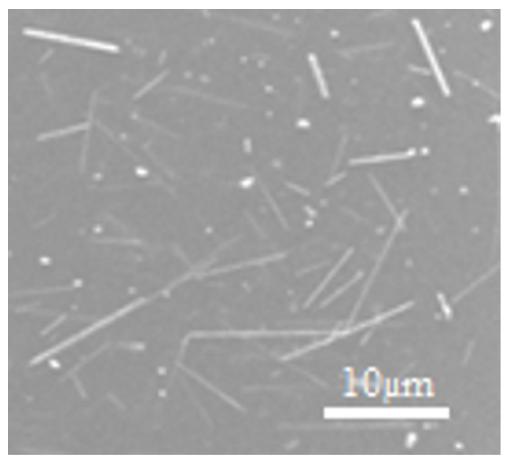

(a)

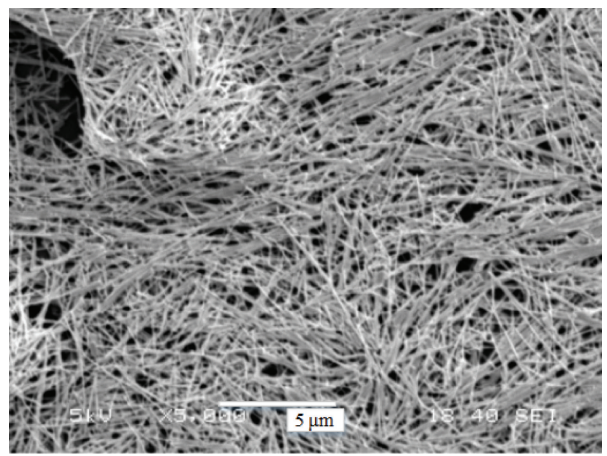

(b)

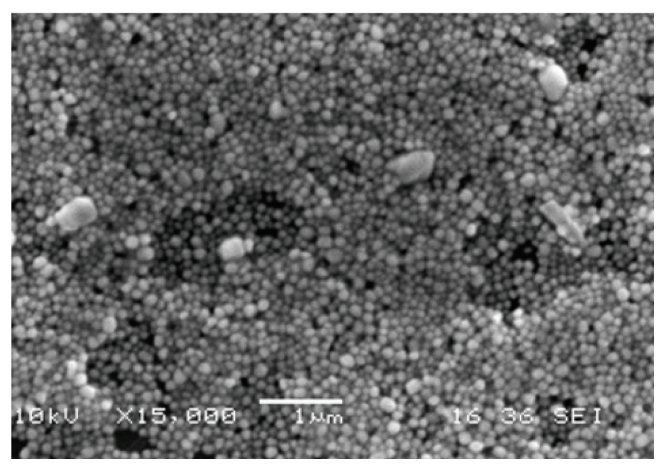

(c)

Figure 2: (a) The image of AgNWs in the solution, (b) centrifuged AgNWs, and (c) AgNPs.

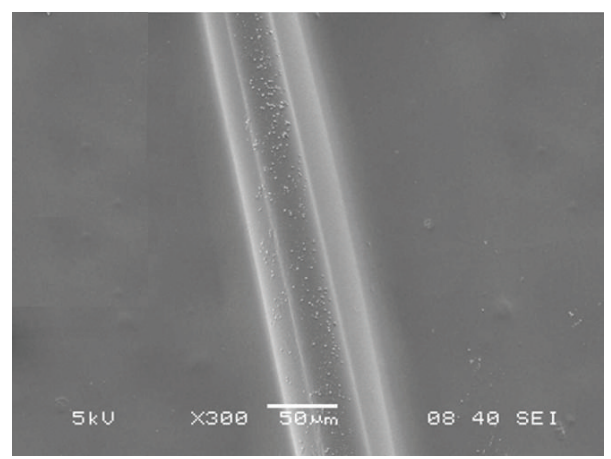

Figure 3: SEM of electrospun AgNPs fiber.

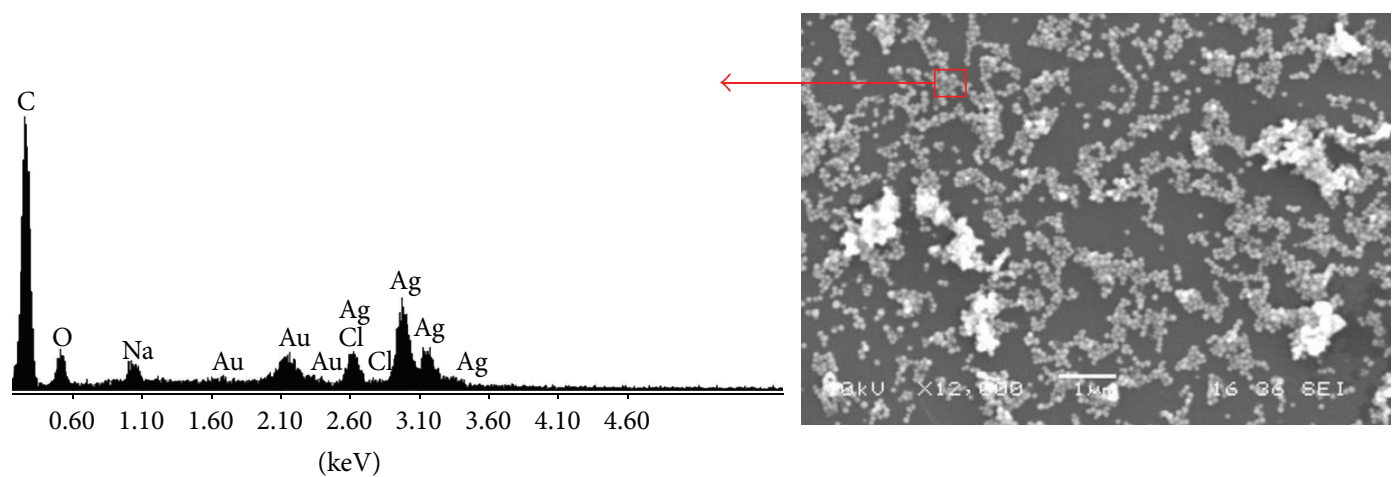

Figure 4: EDS spectrum of AgNPs. 


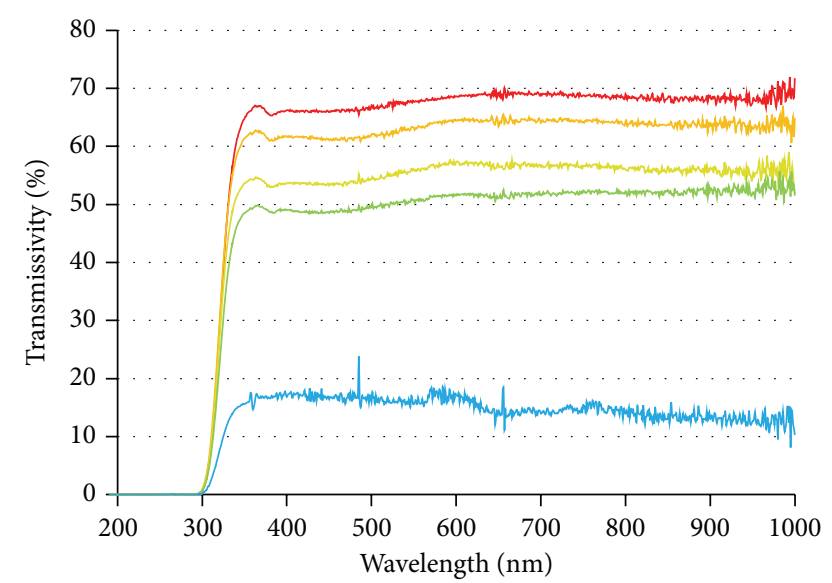

Thickness

$$
\begin{array}{ll}
-304.20 \AA & -705.88 \AA \\
-\quad 482.21 \AA & -2918.0 \AA
\end{array}
$$

Figure 5: Relation between transmissivity and thickness of AgNWs film.

3.3. N\&K Analyzer Measurement. Next, the synthesized AgNWs were used to fabricate AgNWs conductive film whose characteristics were analyzed. The pure AgNWs were used to fabricate AgNWs film. At first, few solutions with evenly dispersed pure AgNWs were taken out by a straw. Next, the solution was dropped on the substrate and then spin coated with different rotational speed to fabricate AgNWs film with different thickness. N\&K analyzer was used to measure the optical properties of the film by using wavelength of 190 1000 nm light source. Figure 5 shows the relation between thickness and transmissivity of AgNWs film. When the thickness of AgNWs films was $31 \mathrm{~nm}, 48 \mathrm{~nm}, 59 \mathrm{~nm}$, $71 \mathrm{~nm}$, and $292 \mathrm{~nm}$, the transmissivity of AgNWs film was $70 \%, 65 \%, 55 \%, 50 \%$, and $15 \%$, respectively. The transmissivity decreased by $5 \%$ with increasing $100 \AA$ thickness of film. Moreover, the average refractive index was 1.20 .

3.4. Analysis of AgNWs Conductive Film. Four-point probe was used to measure sheet resistance by adding constant current between outside two probes and measuring the voltage between inside two probes simultaneously. Figure 6 shows the relation of thickness of film and sheet resistance for five AgNWs films samples: number 1 number 5. The sheet resistance of AgNWs film was $210.8 \Omega / \mathrm{sq}, 210.8 \Omega / \mathrm{sq}$, $2.686 \Omega / \mathrm{sq}, 0.032 \Omega / \mathrm{sq}$, and $0.032 \Omega / \mathrm{sq}$ when the thickness of film was $0.791 \mu \mathrm{m}, 1.123 \mu \mathrm{m}, 1.126 \mu \mathrm{m}, 1.626 \mu \mathrm{m}$, and $1.640 \mu \mathrm{m}$. The increased thickness of film could make sheet resistance decrease; namely, the conductivity could increase. Therefore, the sheet resistance increased as the thickness of AgNWs film decreased.

\section{Conclusion}

The AgNWs and AgNPs solutions were fabricated. Then, the AgNPs fibers were successfully fabricated via the NFES

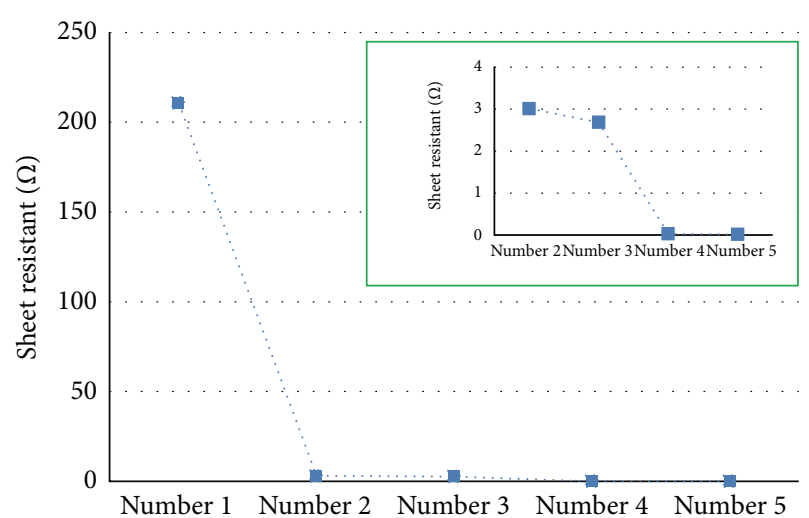

FIGURE 6: Relation between sheet resistance and thickness of AgNWs film.

process with $10 \mathrm{kV}$ applied voltage. The electrospun AgNPs fiber was straight. In this study, the paths and patterns of electrospun AgNPs fiber could be well controlled by the XY programmable table. Next, the AgNWs solution was centrifuged to obtain pure AgNWs which were used to fabricate AgNWs film and the transmissivity and sheet resistance of film were analyzed. The transmissivity of AgNWs film with $31 \mathrm{~nm}$ thickness was $70 \%$ and the sheet resistance of AgNWs film with $1.640 \mu \mathrm{m}$ thickness was $0.032 \Omega$. From the analysis of thickness, sheet resistance, and transmissivity, the transmissivity decreased by $5 \%$ with increasing in film thickness of $100 \AA$ and the sheet resistance increased as the thickness of AgNWs film decreased.

\section{Conflict of Interests}

The authors declare that there is no conflict of interests regarding the publication of this paper.

\section{References}

[1] J. Zou, H.-L. Yip, S. K. Hau, and A. K.-Y. Jen, "Metal grid/conducting polymer hybrid transparent electrode for inverted polymer solar cells," Applied Physics Letters, vol. 96, no. 20, Article ID 203301, pp. 203-301, 2010.

[2] S. Takamatsu, T. Takahata, M. Muraki, E. Iwase, K. Matsumoto, and I. Shimoyama, "Transparent conductive-polymer strain sensors for touch input sheets of flexible displays," Journal of Micromechanics and Microengineering, vol. 20, no. 7, Article ID 075017, 2010.

[3] S. Takamatsu, T. Takahata, K. Matsumoto, and I. Shimoyama, "Micro-patterning of a conductive polymer and an insulation polymer using the Parylene lift-off method for electrochromic displays," Journal of Micromechanics and Microengineering, vol. 21, no. 7, Article ID 075021, 2011.

[4] C. J. M. Emmott, A. Urbina, and J. Nelson, "Environmental and economic assessment of ITO-free electrodes for organic solar cells," Solar Energy Materials and Solar Cells, vol. 97, pp. 14-21, 2012.

[5] Y. Lin, H. F. Dam, T. R. Andersen et al., "Ambient roll-to-roll fabrication of flexible solar cells based on small molecules," 
Journal of Materials Chemistry C, vol. 1, no. 48, pp. 8007-8010, 2013.

[6] H. Park, P. R. Brown, V. Bulović, and J. Kong, "Graphene as transparent conducting electrodes in organic photovoltaics: studies in graphene morphology, hole transporting layers, and counter electrodes," Nano Letters, vol. 12, no. 1, pp. 133-140, 2012.

[7] T. Araki, J. Jiu, M. Nogi et al., "Low haze transparent electrodes and highly conducting air dried films with ultra-long silver nanowires synthesized by one-step polyol method," Nano Research, vol. 7, no. 2, pp. 236-245, 2014.

[8] Z. Yu, Q. Zhang, L. Li et al., "Highly flexible silver nanowire electrodes for shape-memory polymer light-emitting diodes," Advanced Materials, vol. 23, no. 5, pp. 664-668, 2011.

[9] Y. M. Shin, M. M. Hohman, M. P. Brenner, and G. C. Rutledge, "Electrospinning: a whipping fluid jet generates submicron polymer fibers," Applied Physics Letters, vol. 78, no. 8, pp. 11491151, 2001.

[10] D. Sun, C. Chang, S. Li, and L. Lin, "Near-field electrospinning," Nano Letters, vol. 6, no. 4, pp. 839-842, 2006. 

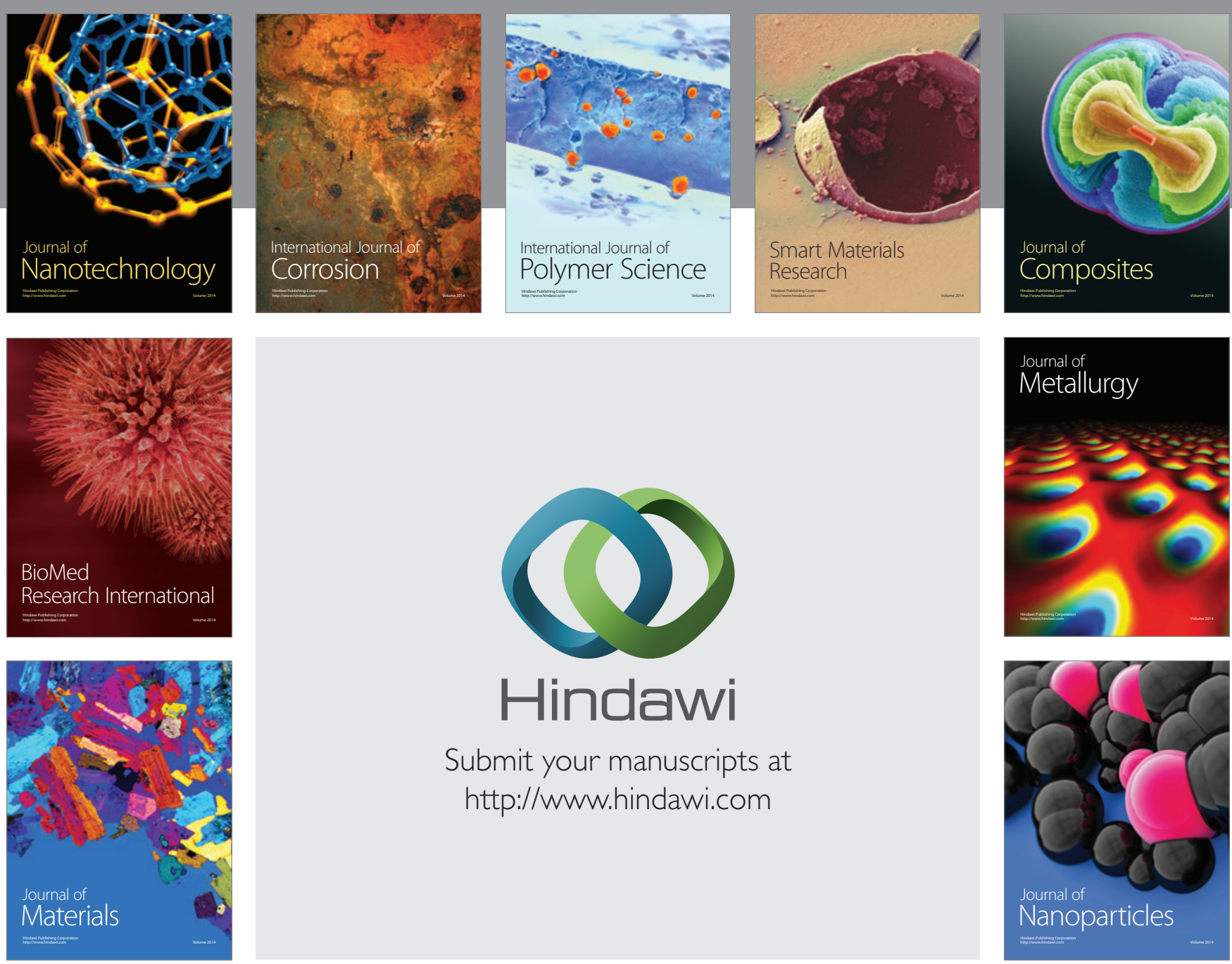

Submit your manuscripts at http://www.hindawi.com
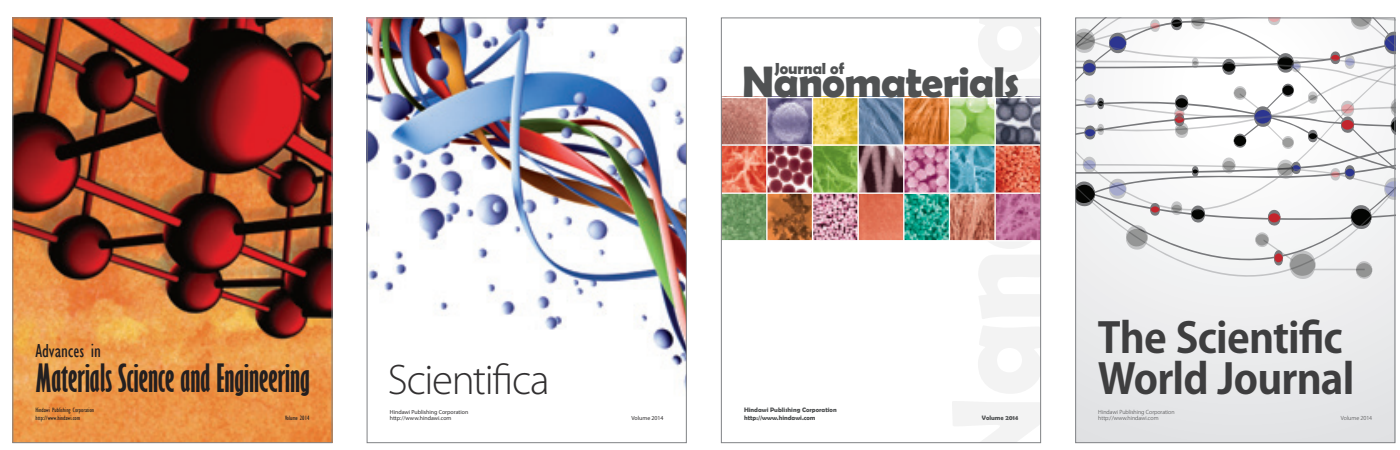

\section{The Scientific World Journal}
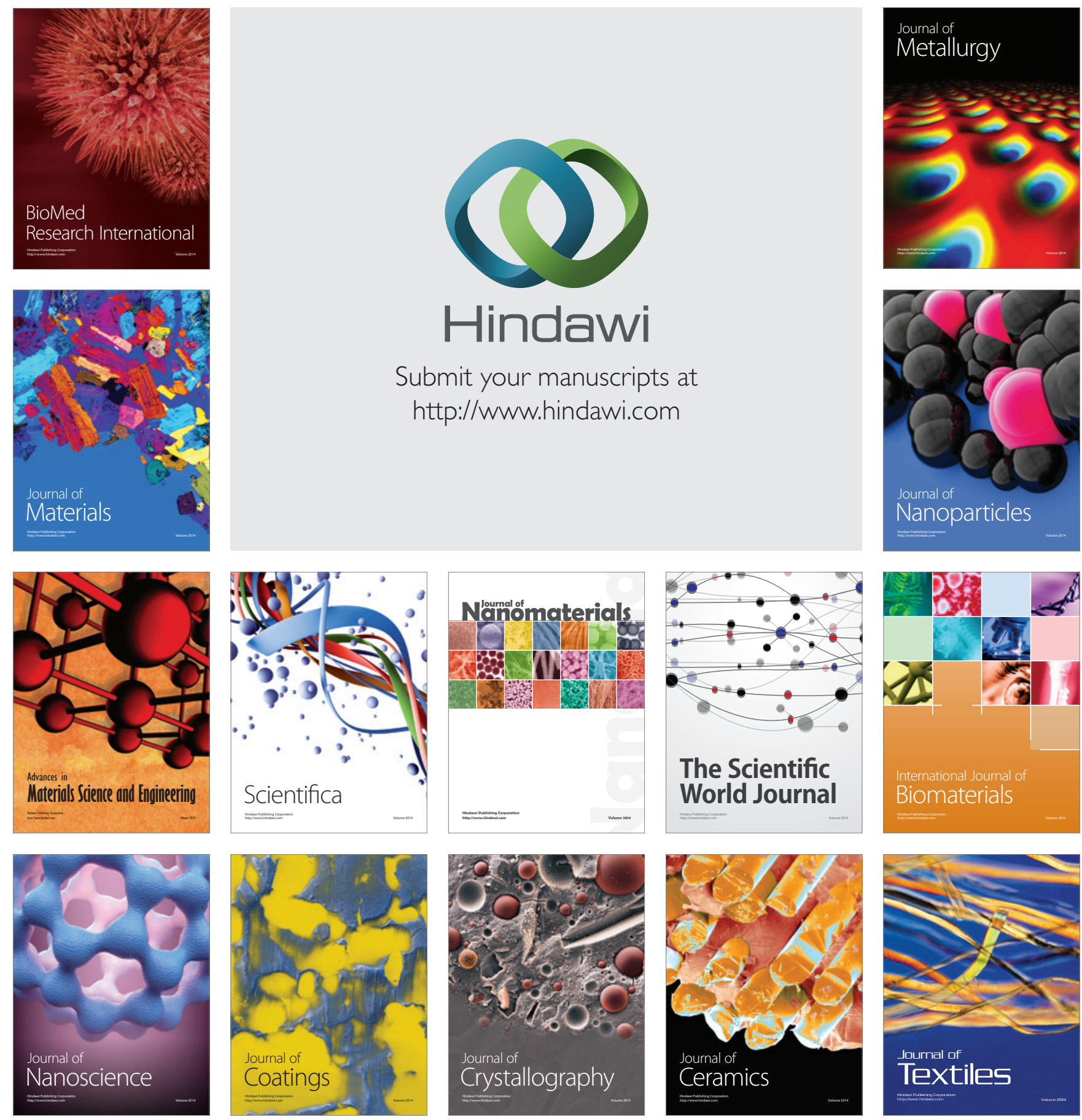